\title{
Intravenous fluid resuscitation is associated with septic endothelial glycocalyx degradation
}

Joseph A. Hippensteel', Ryo Uchimido², Patrick D. Tyler², Ryan C. Burke², Xiaorui Han², Fuming Zhang ${ }^{3}$, Sarah A. McMurtry ${ }^{1}$, James F. Colbert ${ }^{1}$, Christopher J. Lindsell, Derek C. Angus ${ }^{5}$, John A. Kellum ${ }^{4}$, Donald M. Yealy ${ }^{6}$, Robert J. Linhardt ${ }^{3}$, Nathan I. Shapiro ${ }^{2+}$ and Eric P. Schmidt ${ }^{1,7^{*+}}$ (D)

\begin{abstract}
Background: Intravenous fluids, an essential component of sepsis resuscitation, may paradoxically worsen outcomes by exacerbating endothelial injury. Preclinical models suggest that fluid resuscitation degrades the endothelial glycocalyx, a heparan sulfate-enriched structure necessary for vascular homeostasis. We hypothesized that endothelial glycocalyx degradation is associated with the volume of intravenous fluids administered during early sepsis resuscitation.

Methods: We used mass spectrometry to measure plasma heparan sulfate (a highly sensitive and specific index of systemic endothelial glycocalyx degradation) after $6 \mathrm{~h}$ of intravenous fluids in 56 septic shock patients, at presentation and after $24 \mathrm{~h}$ of intravenous fluids in 100 sepsis patients, and in two groups of non-infected patients. We compared plasma heparan sulfate concentrations between sepsis and non-sepsis patients, as well as between sepsis survivors and sepsis non-survivors. We used multivariable linear regression to model the association between volume of intravenous fluids and changes in plasma heparan sulfate.

Results: Consistent with previous studies, median plasma heparan sulfate was elevated in septic shock patients (118 [IQR, 113-341] ng/ml $6 \mathrm{~h}$ after presentation) compared to non-infected controls (61 [45-79] ng/ml), as well as in a second cohort of sepsis patients (283 [155-584] $\mathrm{ng} / \mathrm{ml}$ ) at emergency department presentation) compared to controls (177 [144-262] ng/ml). In the larger sepsis cohort, heparan sulfate predicted in-hospital mortality. In both cohorts, multivariable linear regression adjusting for age and severity of illness demonstrated a significant association between volume of intravenous fluids administered during resuscitation and plasma heparan sulfate. In the second cohort, independent of disease severity and age, each 1 I of intravenous fluids administered was associated with a $200 \mathrm{ng} / \mathrm{ml}$ increase in circulating heparan sulfate $(p=0.006)$ at $24 \mathrm{~h}$ after enrollment.

Conclusions: Glycocalyx degradation occurs in sepsis and septic shock and is associated with in-hospital mortality. The volume of intravenous fluids administered during sepsis resuscitation is independently associated with the degree of glycocalyx degradation. These findings suggest a potential mechanism by which intravenous fluid resuscitation strategies may induce iatrogenic endothelial injury.
\end{abstract}

Keywords: Sepsis, Multiple organ failure, Endothelial glycocalyx, Fluid resuscitation

\footnotetext{
*Correspondence: eric.schmidt@ucdenver.edu

${ }^{\dagger}$ Nathan I. Shapiro and Eric P. Schmidt contributed equally to this work.

${ }^{1}$ Department of Medicine, University of Colorado Denver, Aurora, CO, USA

${ }^{7}$ Department of Medicine, Denver Health Medical Center, Denver, CO, USA

Full list of author information is available at the end of the article
}

(c) The Author(s). 2019 Open Access This article is distributed under the terms of the Creative Commons Attribution 4.0 International License (http://creativecommons.org/licenses/by/4.0/), which permits unrestricted use, distribution, and reproduction in any medium, provided you give appropriate credit to the original author(s) and the source, provide a link to the Creative Commons license, and indicate if changes were made. The Creative Commons Public Domain Dedication waiver (http://creativecommons.org/publicdomain/zero/1.0/) applies to the data made available in this article, unless otherwise stated. 


\section{Background}

Since its introduction during the cholera epidemics of the nineteenth century, intravenous fluid resuscitation has served as a mainstay of supportive sepsis care $[1,2]$. Today, there is increasing concern that intravenous fluids may unexpectedly augment septic endothelial dysfunction, potentially negating the beneficial hemodynamic effects of fluid resuscitation [3]. Such iatrogenic injury could explain the findings of several recent randomized trials which demonstrated that early bolus intravenous fluids worsened sepsis survival $[4,5]$, as well as observational studies that identified associations between fluid administration [6, 7], fluid balance [8-12], and adverse outcomes.

The mechanisms by which intravenous fluid resuscitation could cause harm are uncertain. Preclinical studies suggest that intravenous crystalloids promote degradation of the endothelial glycocalyx [13], a ubiquitous endothelial cellsurface layer composed of transmembrane or membraneanchored proteoglycans (such as syndecan-1) covalently decorated with sulfated glycosaminoglycans (predominantly heparan sulfate, Fig. 1a). The glycocalyx is essential to microvascular homeostasis, as it contributes to the endothelial barrier, mediates shear-induced vasorelaxation, and opposes leukocyte-endothelial adhesion [14]. During sepsis, tumor necrosis factor- $\alpha$ [15] and angiopoietin-2 [16] induce endothelial expression and activation of heparanase, an endoglucuronidase that degrades glycocalyx heparan sulfate, inducing endothelial dysfunction and consequent organ injury. Heparanase and inflammatory stimuli may additionally induce metalloproteinase-mediated shedding of syndecan-1 [17], potentially augmenting glycocalyx collapse. Therefore, the presence of circulating glycocalyx constituents such as heparan sulfate or syndecan-1 fragments indicates a loss of glycocalyx integrity and associated endothelial injury [18].

Preclinical studies have suggested that atrial natriuretic peptide (ANP), a hormone released in response to volume loading-induced atrial stretch, is sufficient to degrade the endothelial glycocalyx in non-septic animals and humans [19-21]. Similarly, a preclinical study of ovine endotoxemia observed that intravenous fluid resuscitation induced a simultaneous rise in circulating glycocalyx fragments and plasma ANP, coincident with worsened septic vasoplegia [13]. These concordant observations suggest that ANP upregulation could be a mechanism for volume overload-related glycocalyx degradation independent of tumor necrosis factor- $\alpha$ and angiopoietin-2-related degradation. The association between ANP and glycocalyx degradation in septic humans, however, has not been explored.

To explore the potential importance of the glycocalyx in human sepsis pathophysiology as well as the association between intravenous fluid resuscitation and glycocalyx degradation, we measured circulating glycocalyx constituents in (a) a subgroup of septic shock patients enrolled in the Protocolized Care for Early Septic Shock (ProCESS) trial and (b) sepsis patients presenting to emergency departments (EDs) at the Beth Israel Deaconess Medical Center (BIDMC, Boston, MA, USA) or St. Vincent's Hospital (Worchester, MA, USA). We hypothesized that (1) the degree of glycocalyx degradation, as measured by circulating heparan sulfate, is associated with sepsis severity and mortality and (2) the volume of intravenous fluids administered early in resuscitation is independently associated with the degree of glycocalyx degradation.

\section{Methods \\ Study populations}

The study population for the ProCESS study [22] and the ProCESS Microcirculatory Flow Ancillary Study [23] has been described in detail elsewhere. In brief, subjects all had (a) suspected infection in the ED, (b) at least two systemic inflammatory response syndrome (SIRS) criteria [24], and (c) refractory hypotension defined as a systolic blood pressure $<90 \mathrm{mmHg}$ despite an IV fluid challenge of at least $1 \mathrm{l}$ crystalloids or evidence of tissue hypoperfusion (blood lactate concentration $\geq 4 \mathrm{mmol} / \mathrm{l}$ ). We used a convenience sample of 56 patients enrolled at sites participating in the ProCESS Microcirculatory Flow Ancillary Study [23] to perform an initial assessment of glycocalyx degradation in sepsis. We measured plasma heparan sulfate in samples collected $6 \mathrm{~h}$ after ProCESS study enrollment, coinciding with the completion of initial volume resuscitation. For comparison, we performed measurements using samples collected from 15 patients presenting with minor, non-infectious complaints to EDs at either BIDMC, Massachusetts General Hospital, or Brigham and Women's Hospital (Boston, MA, USA). In ProCESS patients, we additionally measured levels of circulating syndecan-1 as a second marker of glycocalyx degradation to determine if a more inexpensive ELISAbased assessment of glycocalyx degradation correlated with the "gold standard" of circulating heparan sulfate levels by mass spectrometry.

To validate observations in the ProCESS patients, we enrolled a second group of patients recruited from BIDMC and St. Vincent's Hospital. Patients were adult (age $>18$ years) ED patients presenting with suspected sepsis, enrolled on a convenience basis. We included patients representative of the entire spectrum of sepsis severity, defined by maximum sepsis syndrome severity in the first $72 \mathrm{~h}$ of study enrollment [25]. We selected 100 subjects split roughly evenly between sepsis severities: infected/sepsis patients, severe sepsis (sepsis plus organ dysfunction), and septic shock (sepsis plus systolic blood pressure $<90 \mathrm{mmHg}$ after a minimum of 11 intravenous fluid administration) to comprise our study cohort. Thirty ED patients presenting with minor non- 
A

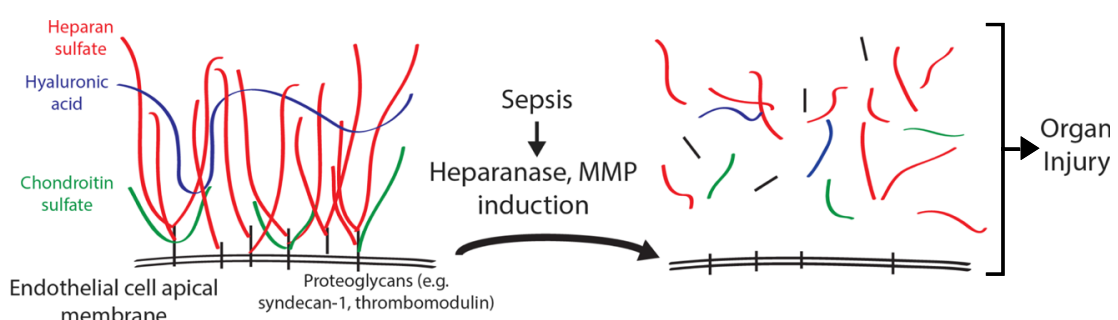

B

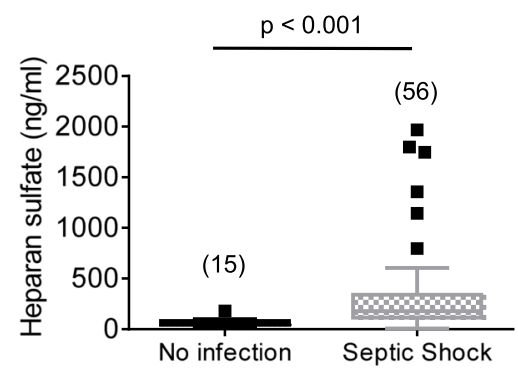

D

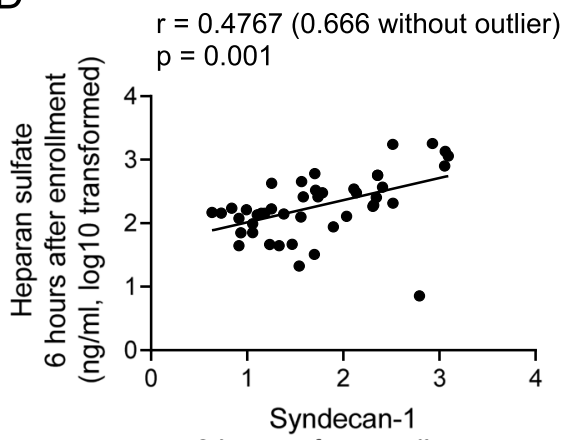

6 hours after enrollment ( $\mu \mathrm{g} / \mathrm{ml}, \log 10$ transformed)

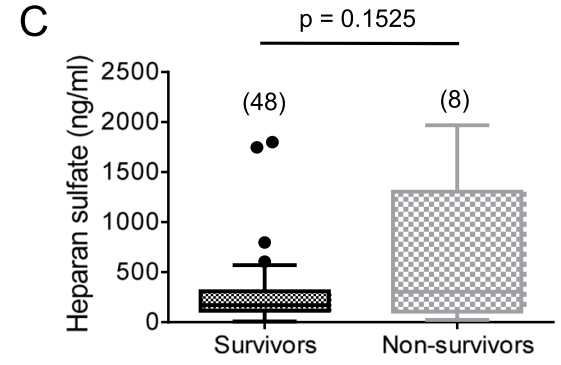

E

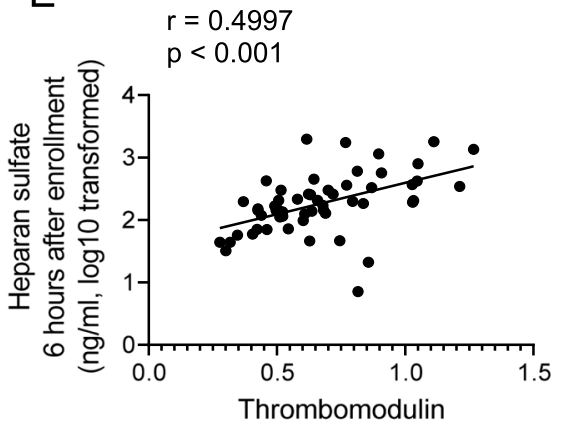

6 hours after enrollment (ng/ml, log10 transformed)

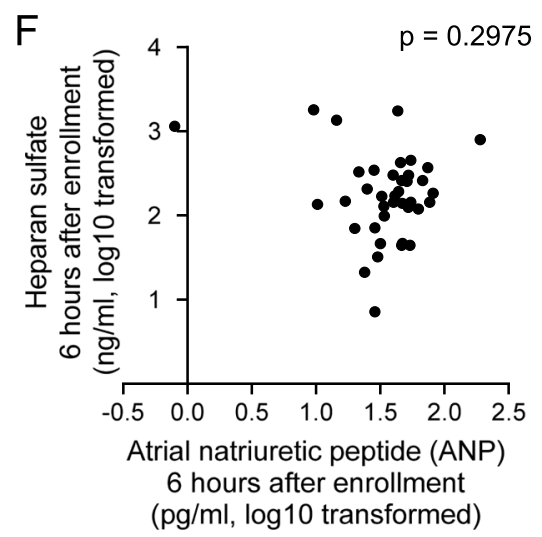

Fig. 1 Glycocalyx degradation occurs in patients with septic shock. a The endothelial glycocalyx is an apical endothelial layer composed of transmembrane proteoglycans (such as syndecan-1 and thrombomodulin) covalently attached to glycosaminoglycans (primarily heparan sulfate) that project into the vascular lumen. During sepsis, activation of heparanase and matrix metalloproteinases leads to glycocalyx degradation, releasing heparan sulfate and proteoglycan fragments into the plasma. $\mathbf{b}$ In a cohort of 56 septic shock patients enrolled in the ProCESS trial, circulating heparan sulfate levels were elevated in comparison to 15 non-infected ED controls. Measurements in septic patients were made $6 \mathrm{~h}$ after enrollment (i.e., after initial fluid resuscitation). c Of 56 septic patients, 8 patients eventually died during their hospitalization. There was a non-significant trend towards increased heparan sulfate concentrations (measured after $6 \mathrm{~h}$ resuscitation) in non-survivors. Circulating heparan sulfate concentrations (at 6 h) correlated with plasma concentrations of glycocalyx components syndecan-1 (d) and thrombomodulin (e) in septic shock patients. Line represents best fit line. $\mathbf{f}$ There was no association between plasma heparan sulfate and atrial natriuretic peptide (ANP) in septic shock patients after $6 \mathrm{~h}$ resuscitation. Parentheses in $\mathbf{b}, \mathbf{c}$ represent number of patients in each group 
infectious complaints served as controls. In this cohort, we collected samples from patients at ED presentation and $24 \mathrm{~h}$ later, and we recorded the volumes of intravenous fluids administered between these time points. All samples were processed within 60 min of being obtained and stored at $-80^{\circ} \mathrm{C}$ until analysis.

The University of Pittsburgh and BIDMC Committees for Clinical Investigations, and the local review boards at each enrolling site approved the study design. Each subject or legal authorized representative gave written informed consent.

\section{Antibodies and reagents}

We measured plasma syndecan-1 (ab46506, Abcam, Cambridge, MA, USA) and brain natriuretic peptide (BNP; ab193694, Abcam) by ELISA, and plasma ANP (RAB0385, Millipore Sigma, St. Louis, MO, USA) by EIA. For the ProCESS cohort, indices of endothelial injury and coagulation including thrombomodulin (an endothelial surface chondroitin sulfate glycosaminoglycan), soluble fms-like tyrosine kinase (sFLT-1; also known as soluble vascular endothelial growth factor receptor-1), angiopoietin 2, and tissue plasminogen activator (tPA) were measured as previously described [26]. Serum lactate and D-dimer were measured as part of the parent ProCESS study. Serum interleukin 6 and tumor necrosis factor- $\alpha$ were measured by ELISA (Quantikine, R\&D Systems, Minneapolis, MN, USA).

\section{Quantification of plasma heparan sulfate}

As previously described, we isolated glycosaminoglycans from EDTA plasma using a spin-column approach [27]. After desalting, we enzymatically digested glycosaminoglycans into component disaccharides. We then 2-aminoacridone-labeled disaccharides and quantified heparan sulfate concentrations using liquid chromatography-mass spectrometry multiple reaction monitoring (LC-MS/MS MRM) [28]. This highly sensitive approach, previously developed [29] and validated [30] by our group, is capable of detecting circulating heparan sulfate of all sulfation types, contrasting the limitation of antibody-based assays to only a few sulfation patterns. We have previously demonstrated that this LC-MS/ MS MRM approach to measuring circulating heparan sulfate is highly sensitive to both septic and non-septic glycocalyx degradation [31] and is an early predictor of glycocalyx degradation-associated organ injury [28].

\section{Statistical analysis}

For the ProCESS microcirculatory flow cohort, we used samples available from the 6-h (post-resuscitation) timepoint to assess differences in levels of circulating heparan sulfate between patients with and without sepsis, and between survivors and non-survivors. We used linear regression to evaluate the association between intravenous fluid volume and heparan sulfate levels, adjusting for age and severity of illness using the Sequential Organ Failure Assessment (SOFA) score at presentation. We considered variables such as demographics (e.g., age, gender) and co-morbidities (e.g., congestive heart failure and chronic kidney disease) for the model and used a forward selection model, allowing variables below the threshold of $p<0.2$ to be eligible to enter the model, and retaining covariates significant at the $p<0.05$ threshold.

We repeated this approach for the BIDMC/St. Vincent's cohort, comparing heparan sulfate levels at ED presentation with maximum sepsis syndrome severity within $72 \mathrm{~h}$. Additionally, we assessed the relationship between the volume of intravenous fluids administered in the $24 \mathrm{~h}$ following ED presentation and the change in circulating heparan sulfate over this time period, using a linear regression model adjusted for age and severity of illness (baseline SOFA score). We repeated the analysis stratified by sepsis syndrome at presentation to further assess the relationship with illness severity.

For both cohorts, we analyzed data using Prism (GraphPad, San Diego, CA, USA) and SAS (Cary, NC, USA) for multivariable analyses. For comparison of two groups, we used a Mann-Whitney test. For comparison of multiple groups, we used Kruskal-Wallis testing with Dunn's post hoc analysis for two-group comparisons. We assessed correlations by Pearson's correlation of logtransformed data. We performed receiver-operating characteristic curves for in-hospital mortality. We share data as box and whisker graphs (demonstrating median, 25th, and 75th percentile data with Tukey representation of outliers) and set the per-comparison alpha error at 0.05 .

\section{Results}

\section{ProCESS patient cohort}

Analyses of plasma samples collected from ProCESS patients ("ProCESS Study", Table 1) after completion of trial-directed fluid resuscitation ( $6 \mathrm{~h}$ after patient enrollment) demonstrated higher levels of circulating heparan sulfate as compared to non-infected ED controls (Fig. 1b). This elevation of circulating heparan sulfate in septic patients is consistent with previous reports [27, 32]. There was a non-statistically significant trend towards increased plasma heparan sulfate in the 8 patients of this cohort who died later in their hospitalization (Fig. 1c), with an area under the ROC curve of $0.661(p=0.1466)$. Plasma heparan sulfate correlated with other measures of glycocalyx degradation, such as the shed proteoglycans syndecan1 (Fig. 1d) and thrombomodulin (Fig. 1e).

Given the known importance of the endothelial glycocalyx to vascular homeostasis, we compared plasma heparan sulfate concentrations with circulating markers of endothelial injury, coagulation, and inflammation (6h after study enrollment). As detailed in Table 2, plasma 
Table 1 Characteristics of two sepsis cohorts

\begin{tabular}{|c|c|c|c|}
\hline \multirow{2}{*}{$\begin{array}{l}\text { Characteristic } \\
\mathrm{n}(\%) \text { unless otherwise noted }\end{array}$} & \multirow[t]{2}{*}{ ProCESS Study $(\mathrm{N}=56)$} & \multicolumn{2}{|c|}{ BIDMC/St. Vincent's Cohort $(\mathrm{N}=130)$} \\
\hline & & Sepsis $(n=100)$ & Control (30) \\
\hline Age - year, ${ }^{b}$ mean $(\mathrm{STD})$ & $59.4( \pm 15.3)$ & $61.4( \pm 2.7)$ & $61.6( \pm 16.1)$ \\
\hline Female sex, $\mathrm{n}(\%)$ & $26(46)$ & $34(34)$ & $12(48)$ \\
\hline \multicolumn{4}{|l|}{ Race, $n(\%)$} \\
\hline White & $43(77)$ & $84(84)$ & $17(68)$ \\
\hline Black or African American & $8(14)$ & $14(14)$ & $7(28)$ \\
\hline Asian & $4(7.1)$ & $1(1.0)$ & $1(4.0)$ \\
\hline Other & $1(1.8)$ & $1(1.0)$ & $0(0.0)$ \\
\hline \multicolumn{4}{|l|}{ Ethnicity n (\%) } \\
\hline Non-Hispanic & $52(93)$ & $100(100)$ & $0(0.0)$ \\
\hline Hispanic & $4(7.1)$ & $0(0.0)$ & $1(4.0)$ \\
\hline \multicolumn{4}{|l|}{ Chronic conditions n (\%) unless specified } \\
\hline Charlson comorbidity score, mean (STD) & $2.7( \pm 2.7)$ & $3.7( \pm 2.7)$ & $3.0( \pm 2.2)$ \\
\hline Hypertension & $29(52)$ & $49(49)$ & $11(44)$ \\
\hline Diabetes mellitus & $16(29)$ & $35(35)$ & $9(36)$ \\
\hline Chronic respiratory disease & $14(25)$ & $13(13)$ & $1(4.0)$ \\
\hline Cancer & $14(25)$ & $11(11)$ & $1(4.0)$ \\
\hline Dialysis dependent Renal impairment & $2(3.6)$ & $0(0.0)$ & $0(0.0)$ \\
\hline Congestive heart failure & $10(18)$ & $17(17)$ & $3(12)$ \\
\hline Prior myocardial infarction & $2(3.6)$ & $3(3.0)$ & $1(4.0)$ \\
\hline Cerebral vascular disease & $6(11)$ & $3(3.0)$ & $1(4.0)$ \\
\hline Peripheral vascular disease & $5(8.9)$ & $7(7.0)$ & $2(8.0)$ \\
\hline Chronic dementia & $2(3.6)$ & $0(0.0)$ & $0(0.0)$ \\
\hline Hepatic cirrhosis & $0(0)$ & $0(0.0)$ & $0(0.0)$ \\
\hline Peptic ulcer disease & $1(1.8)$ & $1(1.0)$ & $0(0.0)$ \\
\hline AIDS and related syndromes & $0(0.0)$ & $3(3.0)$ & $1(4.0)$ \\
\hline Baseline SOFA Score & $7.0( \pm 4.1)$ & $3.1( \pm 2.9)$ & $\mathrm{N} / \mathrm{A}$ \\
\hline \multicolumn{4}{|l|}{ Hemodynamic Variable, mean (STD) } \\
\hline Systolic Blood Pressure & $93(15)$ & $136(16)$ & $116(27)$ \\
\hline Mean Arterial Pressure & $68(11)$ & $94(14)$ & $74(20)$ \\
\hline Heart Rate & $101(20)$ & $73(24)$ & $98(16)$ \\
\hline \multicolumn{4}{|l|}{ Worst sepsis syndrome } \\
\hline Infection/Sepsis & $0(0.0)$ & $28(28)$ & N/A \\
\hline Severe sepsis & $0(0.0)$ & $41(41)$ & N/A \\
\hline Septic shock & $56(100)$ & $31(31)$ & N/A \\
\hline Total Fluids Administered in mL (STD) & $2578( \pm 1622) 0-6 h$ & $1681( \pm 1801) 0-24 \mathrm{~h}$ & N/A \\
\hline Crystalloid in $\mathrm{mL}$ (STD) & $2389( \pm 1573) 0-6 h$ & $1667( \pm 1879) 0-24 \mathrm{~h}$ & N/A \\
\hline Colloid in $\mathrm{mL}$ (STD) & $7( \pm 53) 0-6 \mathrm{~h}$ & $11( \pm 62) 0-24 \mathrm{~h}$ & N/A \\
\hline Blood Products in mL (STD) & $182( \pm 500) 0-6 \mathrm{~h}$ & $3.5( \pm 35) 0-24 \mathrm{~h}$ & N/A \\
\hline
\end{tabular}

Table 2 Associations of circulating heparan sulfate with plasma indices of endothelial injury, coagulation, and inflammation (ProCESS cohort, $6 \mathrm{~h}$ after enrollment)

\begin{tabular}{|c|c|c|c|}
\hline Marker & Pearson $r$ & $p$-value & Number of subjects analyzed \\
\hline \multicolumn{4}{|l|}{ Endothelial Injury/Activation } \\
\hline sFLT-1 (soluble VEGF-receptor 1) & 0.4097 & 0.0017 & 56 \\
\hline Angiopoietin-2 & 0.2367 & 0.0790 & 56 \\
\hline \multicolumn{4}{|l|}{ Inflammation } \\
\hline Tumor necrosis factor-a & 0.2325 & 0.1384 & 42 \\
\hline Interleukin-6 & 0.3537 & 0.0216 & 42 \\
\hline \multicolumn{4}{|l|}{ Coagulation } \\
\hline Tissue plasminogen activator & 0.3356 & 0.0114 & 56 \\
\hline D-dimer & 0.0926 & 0.5699 & 40 \\
\hline Thrombomodulin & 0.4997 & $<0.0001$ & 56 \\
\hline \multicolumn{4}{|l|}{ Other } \\
\hline Lactate & -0.0765 & 0.6527 & 37 \\
\hline
\end{tabular}


heparan sulfate was significantly associated with the endothelial activation marker sFLT-1 (with a non-significant trend towards association with angiopoietin-2), the inflammatory marker interleukin-6, and the endogenous thrombolytic tPA. No associations were seen between plasma heparan sulfate and lactate, tumor necrosis factor- $\alpha$, or D-dimer $6 \mathrm{~h}$ after study enrollment.

Interestingly, we observed no association in the ProCESS cohort between plasma heparan sulfate and ANP (Fig. 1f), a hypothesized mediator of fluid-induced glycocalyx shedding [13]. Surprisingly, ANP levels $6 \mathrm{~h}$ after enrollment were elevated in patients who went on to survive septic shock $(47.1 \pm 5.3 \mathrm{pg} / \mathrm{ml}, n=33)$, as compared to those who later died during their hospitalization $(27.0 \pm 7.8 \mathrm{pg} / \mathrm{ml}, n=8, p=0.02$ by Mann-Whitney, area under ROC curve 0.7749). This lack of an association with increased mortality suggests that ANP is not a primary mediator of organ-injurious glycocalyx degradation. We observed no association between IL-6 and ANP $(r=0.09 ; p=0.63, n=29)$, contrasting previous literature implicating IL-6-mediated inflammation (and not volume overload) as the primary trigger for ANP release [33]. Finally, there was no association between brain natriuretic peptide (BNP, a natriuretic peptide also associated with fluid overload) and heparan sulfate $(p=0.367, r=$ $0.148, n=39$ ).

Using multivariable linear regression, we observed that plasma heparan sulfate at the end of sepsis resuscitation (6 $\mathrm{h}$ after enrollment) was associated with the volume of intravenous fluids administered during that resuscitation period, even when adjusting for age and severity of illness (Table 3).

Beth Israel Deaconess Medical Center/St. Vincent's cohort We analyzed plasma collected from an independent cohort of 100 septic patients (defined by Sepsis-2 criteria) at the time of presentation to the EDs of the BIDMC and St. Vincent's Hospital ("BIDMC/St. Vincent's cohort", Table 1) to confirm the generalizability of these initial findings beyond our sampling of ProCESS patients. In addition, 30 non-infected ED patients served as controls. In keeping with the ProCESS cohort, we found an

Table 3 Heparan sulfate shedding $6 \mathrm{~h}$ after ProCESS enrollment is independently associated with the volume of fluid resuscitation received over those $6 \mathrm{~h}(n=56)$

\begin{tabular}{llll}
\hline \multicolumn{4}{l}{ Plasma heparan sulfate (measured at 6 hours after study enrollment) } \\
\hline Variable & Parameter estimate & Standard error & $p$ \\
\hline Intercept & -132.27 & 276.3 & 0.63 \\
SOFA & 25.28 & 14.65 & 0.09 \\
Age & 1.53 & 4.04 & 0.71 \\
$\begin{array}{l}\text { Cumulative intravenous } \\
\text { fluids, 0-6h }\end{array}$ & 0.08 & 0.04 & 0.047 \\
\hline
\end{tabular}

elevation in circulating heparan sulfate levels (at time of ED arrival) in patients who were diagnosed with severe sepsis or septic shock within $72 \mathrm{~h}$ (Fig. 2a). In this cohort, circulating heparan sulfate concentrations at ED presentation were significantly associated with severity of illness (SOFA) at that time $(r=0.4135, p<0.0001$, Fig. 2b). Plasma heparan sulfate at ED arrival was significantly higher in non-survivors, as compared to survivors $(p<$ 0.05 , Fig. 2c, d). Intriguingly, heparan sulfate shedding was more predominant in septic patients with positive blood cultures (Fig. 2e) than blood culture-negative sepsis; no differences were seen between gram positive or gram negative bacteremia.

Ninety-seven of the 100 patients in the BIDMC/St. Vincent's cohort had serial blood draws collected at $0 \mathrm{~h}$ and $24 \mathrm{~h}$ available for analysis. In these patients, the volume of intravenous fluids administered over the first $24 \mathrm{~h}$ of the study correlated with the change in plasma heparan sulfate across that time period, adjusted for age and baseline SOFA (Table 4).

We used paired data from the BIDMC/St. Vincent's sepsis cohort to model the 24-h change in plasma heparan sulfate from intravenous fluid volume administered across that time period, adjusted for age, baseline SOFA score, and $72 \mathrm{~h}$ sepsis syndrome severity. Predicted values are shown in Fig. 3, demonstrating that independent of disease severity and age, each 11 of fluids administered was associated with a $200 \mathrm{ng} / \mathrm{ml}$ increase in circulating heparan sulfate $(p=0.006)$.

\section{Discussion}

Our report supports preclinical observations [13] that both sepsis severity and the volume of intravenous fluids administered during sepsis resuscitation are associated with glycocalyx degradation. While our study design is unable to prove causality, the associations are consistent across patient cohorts. An injurious effect of fluid resuscitation on the endothelial glycocalyx would be expected to worsen sepsis outcomes, given the importance of glycocalyx integrity to vascular homeostasis. In healthy vessels, the intact endothelial glycocalyx functions to oppose transvascular fluid flux and leukocyte adhesion. Accordingly, pathologic loss of glycocalyx integrity during sepsis might directly contribute to the tissue edema characteristic of septic organ injury [14]. Furthermore, as the glycocalyx plays a critical role in regulating nitric oxide synthesis, septic glycocalyx degradation may contribute to the microvascular heterogeneity and vasoplegia characteristic of septic shock [13]. Exacerbation of sepsis-induced glycocalyx degradation by intravenous fluids would therefore be expected to worsen inflammatory organ injury and microcirculatory dysfunction, significantly impacting patient outcomes. 
A

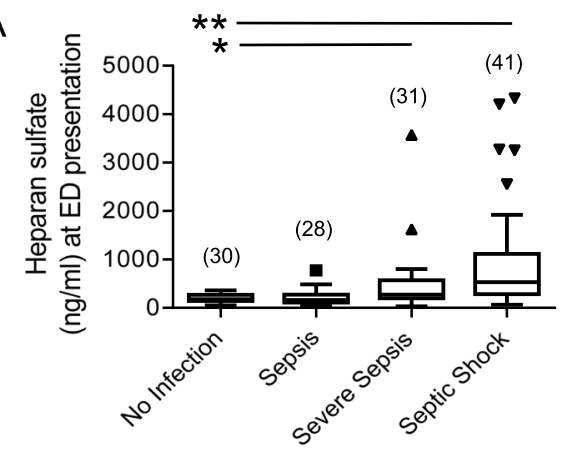

C

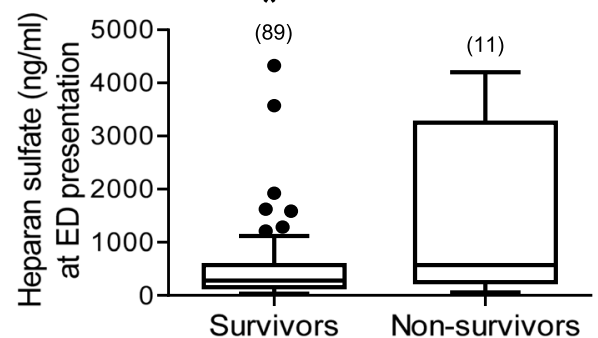

Survivors Non-survivors

E

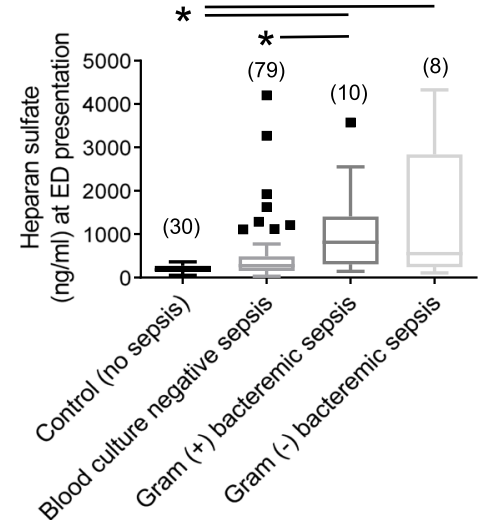

B

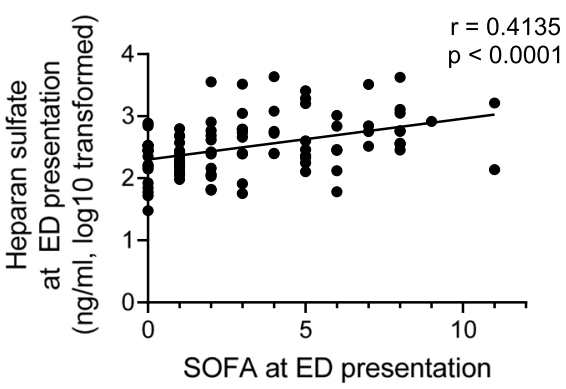

D Heparan sulfate $(\mathrm{ng} / \mathrm{ml})$ at ED presentation as a predictor of mortality

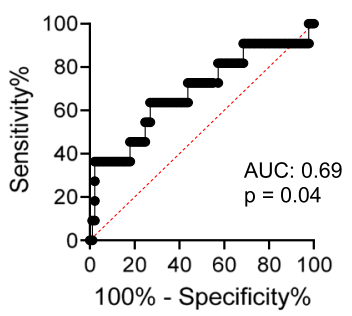

Fig. 2 Circulating glycocalyx degradation products predict clinically relevant outcomes in sepsis patients presenting to the Beth Israel Deaconess Medical Center or St. Vincent's Hospital Emergency Departments. a Elevated levels of circulating heparan sulfate at emergency department (ED) presentation were associated with a diagnosis of severe sepsis or septic shock within the ensuing $72 \mathrm{~h}(n=100)$. b Heparan sulfate levels correlated with increased severity of illness (SOFA) at the time of ED presentation $(n=100)$. c Measures of circulating heparan sulfate in septic patients at ED presentation ( $n=100$ ) were significantly associated with mortality. $\mathbf{d}$ Receiver operating characteristic (ROC) curve for plasma heparan sulfate (at ED presentation) as a predictor of later in-hospital mortality. e Heparan sulfate plasma concentrations at ED presentation were elevated in septic patients with positive bacterial blood cultures. Three blood samples that grew Staph. epidermidis (contaminant) were excluded. ${ }^{*} p<0.05 ;{ }^{*} p<0.0001$. Parentheses represent number of patients

Table 4 In the Beth Israel Deaconess Medical Center/St. Vincent's cohort (100 patients), the 24-h increase in circulating heparan sulfate (an index of ongoing glycocalyx degradation) is independently associated with the volume of fluid resuscitation received over those $24 \mathrm{~h}$

\begin{tabular}{llll}
\hline Change in plasma heparan sulfate (from time of study entry to 24 hours after enrollment) & & Standard error \\
\hline Variable & Parameter estimate & 276.91 & $p$ \\
\hline Intercept & -340.19 & 23.15 & 0.22 \\
SOFA & -13.44 & 4.26 & 0.56 \\
Age & 6.50 & 0.03 & 0.13 \\
Cumulative intravenous fluids, 0-24h & 0.08 & 0.02 \\
\hline
\end{tabular}




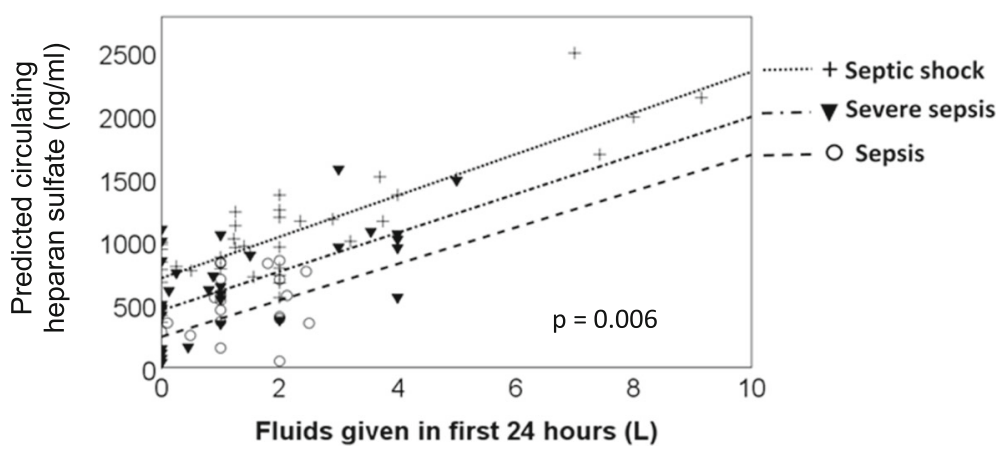

Fig. 3 Volume of intravenous fluids administered early in sepsis predicts degree of glycocalyx degradation. The total volume of intravenous fluids administered in the first $24 \mathrm{~h}$ after ED presentation predicted the change in plasma heparan sulfate levels from initial blood draw to $24 \mathrm{~h}$ blood draw. Model $R^{2}=0.149$, fit lines are shown for patients experiencing sepsis, severe sepsis, and septic shock, adjusted for age and baseline SOFA score

The mechanisms by which intravenous fluids could induce glycocalyx degradation are uncertain. Pre-clinical animal and human studies have shown that ANP degrades the endothelial glycocalyx [19-21], suggesting that fluid resuscitation may cause iatrogenic glycocalyx degradation in septic patients via the induction of volume overload. While our study does support an association between the volume of fluid resuscitation and glycocalyx degradation, we did not observe an association between plasma ANP (or BNP) and heparan sulfate. These findings, while observational and limited to plasma samples available from ProCESS cohort, do not support the hypothesis that natriuretic peptide-mediated degradation in response to volume overload is the primary mechanism responsible for intravenous fluid-associated glycocalyx degradation. Rather, intravenous fluids may be capable of directly inducing endothelial injury and endothelial shedding independently of fluid balance. One possible mechanism is fluctuations in endothelial shear stress caused by fluids. Sudden vascular stretch from fluid boluses paired with the presence of inflammatory mediators may stimulate endothelial expression of glycocalyx-shedding matrix metalloproteinases [34]. Furthermore, oscillatory shear stress may promote cathep$\sin \mathrm{L}$ activation, an enzyme implicated in post-translational activation of endothelial heparanase [35]. Alternatively, isotonic fluid administration could directly activate circulating leukocytes $[36,37]$, potentially inducing neutrophil elastase-mediated glycocalyx degradation [38]. These speculative mechanisms will require additional translational investigation.

Intriguingly, the ProCESS and BIDMC/St. Vincent's cohorts relied upon intravenous saline as the primary volume resuscitation agent, accounting for greater than 90\% of the fluid administered in both cohorts. Emerging clinical and preclinical studies suggest that saline may be injurious when compared to balanced crystalloids [39], albumin [40], and fresh frozen plasma [41] in the resuscitation of critical illness. Future human studies will be necessary to determine if these resuscitation agents are differentially associated with glycocalyx degradation.

Our study has several strengths, including the use of two independent patient cohorts. Additionally, our findings are based upon state-of-the-art mass spectrometry analyses (LC-MS/MS MRM) of plasma heparan sulfate [28], an approach that is highly sensitive to both septic and non-septic endothelial glycocalyx degradation [31]. Circulating heparan sulfate is also highly specific to endothelial glycocalyx degradation: while heparan sulfate exists external to the vascular lumen (e.g. in the basement membrane and interstitium), negatively-charged fragments produced by degradation of extravascular heparan sulfates would be repelled by the negative charge of an intact glycocalyx, preventing plasma penetration. As such, the presence of circulating heparan sulfate fragments necessitates a breach of glycocalyx integrity. The validity of mass spectrometry measures of heparan sulfate as an index of glycocalyx degradation in our cohort is supported by the observed correlation with shed syndecan-1 (Fig. 1d), a commonly-used ELISA-based assay of glycocalyx degradation [18, 42], as well as shed thrombomodulin (Fig. 1e), an endothelial-surface chondroitin sulfate proteoglycan.

Our study also has several limitations. First, we only included a convenience sample of patients in the study cohorts and it is possible that this introduced a selection bias. Second, despite our study being amongst the largest to employ mass spectrometry to investigate glycocalyx degradation in humans, the overall sample size and number of deaths is still relatively low; thus, larger studies are needed to validate our findings. Third, it is possible that other measures of glycocalyx degradation are needed above and beyond circulating levels of heparan sulfate to have a comprehensive readout of glycocalyx degradation. Finally, it is important to emphasize that despite our use 
of multivariable modeling to account for measured confounders, our cohorts were underpowered to address additional pertinent variables that could affect glycocalyx integrity, such as underlying comorbidities [43], use of stress-dose glucocorticoids [44], and appropriate antibiotic choices (Fig. 2e). Our observational study is therefore unable to exclude the contribution of these and other unrecognized confounders of the observed association between fluid resuscitation and glycocalyx degradation. As such, we are unable to conclude causality.

Our observational findings therefore require confirmation by prospective, randomized studies. The Prevention and Treatment of Acute Lung Injury (PETAL) Network has recently initiated a large study of volume resuscitation practices in sepsis. This Crystalloid Liberal or Vasopressors Early Resuscitation in Sepsis (CLOVERS) study will compare the use of a liberal fluids protocol (larger volume of fluids prior to the initiation of vasopressors) with a restrictive fluids protocol (smaller amounts of intravenous fluids and early use of vasopressors) in patients with sepsis-induced hypotension [45]. Studies such as CLOVERS will provide opportunities to leverage randomized assignment of resuscitation strategies, allowing for better insight into the causal relationship between intravenous fluids and glycocalyx degradation.

\section{Conclusions}

Our report demonstrates an association between fluid resuscitation and glycocalyx degradation in sepsis, supporting observations made using preclinical models of endotoxemia [13]. Future randomized controlled studies will provide an opportunity to confirm a causal association.

\section{Abbreviations}

ANP: Atrial natriuretic protein; BIDMC: Beth Israel Deaconness Medical Center; BNP: Brain natriuretic peptide; CLOVERS: Crystalloid Liberal or Vasopressors Early Resuscitation in Sepsis; ED: Emergency department; LC-MS/MS MRM: Liquid chromatography-mass spectrometry multiple reaction monitoring; PETAL: Prevention and Treatment of Acute Lung Injury; ProCESS: Protocolized Care for Early Septic Shock; sFLT-1: Soluble fms-like tyrosine kinase-1; SIRS: Systemic inflammatory response syndrome; SOFA: Sequential Organ Failure Assessment; tPA: Tissue plasminogen activator

\section{Authors' contributions}

JAH, CJL, JFC, RJL, NIS and EPS designed the study, performed statistical analyses, interpreted the data, and wrote the manuscript. RU, PT and RCB performed statistical analyses and assisted with manuscript preparation. DCA, JK and DMY participated in study design and oversaw enrollment of study subjects. XH and FZ designed and performed analyses of glycosaminoglycan content. SAM performed protein analyses. All authors read and approved the final manuscript.

\section{Funding}

R01 HL125371 (to EPS, RJL).

\section{Availability of data and materials}

The datasets generated and/or analyzed during the current study are available from the corresponding author on reasonable request.

\section{Ethics approval and consent to participate}

The University of Pittsburgh and BIDMC Committees for Clinical Investigations, and the local review boards at each enrolling site approved the study design. Each subject or legal authorized representative gave written informed consent.

\section{Consent for publication}

Not applicable.

\section{Competing interests}

The authors declare that they have no competing interests.

\section{Author details}

${ }^{1}$ Department of Medicine, University of Colorado Denver, Aurora, CO, USA. ${ }^{2}$ Department of Emergency Medicine, Beth Israel Deaconess Medical Center, Boston, MA, USA. ${ }^{3}$ Departments of Chemistry and Chemical Biology,

Chemical and Biological Engineering, and Biomedical Engineering, Rensselaer Polytechnic Institute, Troy, NY, USA. ${ }^{4}$ Department of Biostatistics, Vanderbilt University Medical Center, Nashville, TN, USA. ${ }^{5}$ Department of Critical Care Medicine, University of Pittsburgh, Pittsburgh, PA, USA. ${ }^{6}$ Department of Emergency Medicine, University of Pittsburgh, Pittsburgh, PA, USA.

${ }^{7}$ Department of Medicine, Denver Health Medical Center, Denver, CO, USA.

Received: 3 April 2019 Accepted: 1 July 2019

Published online: 23 July 2019

\section{References}

1. MacGillivray N. Dr Thomas Latta: the father of intravenous infusion therapy. J Infect Prev. 2009;10(1_suppl):S3-6.

2. Byrne $L$, Haren F. Fluid resuscitation in human sepsis: time to rewrite history? Ann Intensive Care. 2017;7(1):4.

3. Hippensteel JA, Shapiro NI, Schmidt EP. Challenging dogma: the value of bolus fluids in the early resuscitation of Hyperdynamic Sepsis. Am J Respir Crit Care Med. 2018;198(8):981-3.

4. Maitland K, Kiguli S, Opoka RO, Engoru C, Olupot-Olupot P, Akech SO, Nyeko R, Mtove G, Reyburn H, Lang T, et al. Mortality after fluid bolus in African children with severe infection. N Engl J Med. 2011;364(26):2483-95.

5. Andrews B, Semler MW, Muchemwa L, Kelly P, Lakhi S, Heimburger DC, Mabula C, Bwalya M, Bernard GR. Effect of an early resuscitation protocol on in-hospital mortality among adults with Sepsis and hypotension: a randomized clinical trial. Jama. 2017;318(13):1233-40.

6. Silversides JA, Major E, Ferguson AJ, Mann EE, McAuley DF, Marshall JC, Blackwood B, Fan E. Conservative fluid management or deresuscitation for patients with sepsis or acute respiratory distress syndrome following the resuscitation phase of critical illness: a systematic review and meta-analysis. Intensive Care Med. 2017:43(2):155-70.

7. Marik PE, Linde-Zwirble WT, Bittner EA, Sahatjian J, Hansell D. Fluid administration in severe sepsis and septic shock, patterns and outcomes: an analysis of a large national database. Intensive Care Med. 2017;43(5):625-32.

8. Acheampong A, Vincent J-L. A positive fluid balance is an independent prognostic factor in patients with sepsis. Crit Care. 2015;19(1):251.

9. Boyd JH, Forbes J, T-a N, Walley KR, Russell JA. Fluid resuscitation in septic shock: a positive fluid balance and elevated central venous pressure are associated with increased mortality. Crit Care Med. 2011;39(2):259-65.

10. Sadaka F, Juarez M, Naydenov S, O'brien J. Fluid resuscitation in septic shock: the effect of increasing fluid balance on mortality. J Intensive Care Med. 2014:29(4):213-7.

11. Vincent JL, Sakr Y, Sprung CL, Ranieri VM, Reinhart K, Gerlach H, Moreno R, Carlet J, Le Gall JR, Payen D. Sepsis in European intensive care units: results of the SOAP study. Crit Care Med. 2006;34(2):344-53.

12. Pittard MG, Huang SJ, McLean AS, Orde SR. Association of positive fluid balance and mortality in sepsis and septic shock in an Australian cohort. Anaesth Intensive Care. 2017;45(6):737-43.

13. Byrne L, Obonyo NG, Diab SD, Dunster KR, Passmore MR, Boon AC, See Hoe L, Pedersen S, Hashairi Fauzi M, Pretti Pimenta L, et al. Unintended consequences; fluid resuscitation worsens shock in an ovine model of Endotoxemia. Am J Respir Crit Care Med. 2018;198(8):1043-54.

14. Uchimado R SE, Shapiro NI. The Glyocalyx: a novel diagnostic and therapeutic target in sepsis. Crit Care. 2019:23.

15. Schmidt EP, Yang Y, Janssen WJ, Gandjeva A, Perez MJ, Barthel L, Zemans RL, Bowman JC, Koyanagi DE, Yunt ZX, et al. The pulmonary endothelial 
glycocalyx regulates neutrophil adhesion and lung injury during experimental sepsis. Nat Med. 2012;18(8):1217-23.

16. Han S, Lee SJ, Kim KE, Lee HS, Oh N, Park I, Ko E, Oh SJ, Lee YS, Kim D, et al. Amelioration of sepsis by TIE2 activation-induced vascular protection. Sci Transl Med. 2016;8(335):335ra355.

17. Purushothaman A, Uyama T, Kobayashi F, Yamada S, Sugahara K, Rapraeger AC, Sanderson RD. Heparanase-enhanced shedding of syndecan-1 by myeloma cells promotes endothelial invasion and angiogenesis. Blood. 2010;115(12):2449-57.

18. Colbert JF, Schmidt EP. Endothelial and microcirculatory function and dysfunction in Sepsis. Clin Chest Med. 2016;37(2):263-75.

19. Jacob M, Saller T, Chappell D, Rehm M, Welsch U, Becker BF. Physiological levels of A-, B- and C-type natriuretic peptide shed the endothelial glycocalyx and enhance vascular permeability. Basic Res Cardiol. 2013;108(3):347.

20. Bruegger D, Jacob M, Rehm M, Loetsch M, Welsch U, Conzen P, Becker BF. Atrial natriuretic peptide induces shedding of endothelial glycocalyx in coronary vascular bed of Guinea pig hearts. Am J Physiol Heart Circ Physiol. 2005;289(5):H1993-9.

21. Chappell D, Bruegger D, Potzel J, Jacob M, Brettner F, Vogeser M, Conzen P, Becker BF, Rehm M. Hypervolemia increases release of atrial natriuretic peptide and shedding of the endothelial glycocalyx. Crit Care. 2014;18(5):538.

22. Investigators TP. A randomized trial of protocol-based care for early septic shock. N Engl J Med. 2014;370(18):1683-93.

23. Massey MJ, Hou PC, Filbin M, Wang H, Ngo L, Huang DT, Aird WC, Novack $\checkmark$, Trzeciak S, Yealy DM, et al. Microcirculatory perfusion disturbances in septic shock: results from the ProCESS trial. Crit Care. 2018;22(1):308.

24. Bone R. American College of Chest Physicians/Society of Critical Care Medicine consensus conference: definitions for sepsis and organ failure and guidelines for the use of innovative therapies in sepsis. Crit Care Med. 1992; 20:864-74.

25. Vincent JL, Moreno R, Takala J, Willatts S, De Mendonca A, Bruining H, Reinhart CK, Suter PM, Thijs LG. The SOFA (Sepsis-related organ failure assessment) score to describe organ dysfunction/failure. On behalf of the working group on Sepsis-related problems of the European Society of Intensive Care Medicine. Intensive Care Med. 1996;22(7):707-10.

26. Hou PC, Filbin MR, Wang H, Ngo L, Huang DT, Aird WC, Yealy DM, Angus DC, Kellum JA, Shapiro NI. Endothelial permeability and hemostasis in septic shock: results from the ProCESS trial. Chest. 2017;152(1):22-31.

27. Schmidt EP, Li G, Li L, Fu L, Yang Y, Overdier KH, Douglas IS, Linhardt RJ. The circulating glycosaminoglycan signature of respiratory failure in critically ill adults. J Biol Chem. 2014;289(12):8194-202.

28. Schmidt EP, Overdier KH, Sun X, Lin L, Liu X, Yang Y, Ammons LA, Hiller TD, Suflita MA, Yu Y. Urinary glycosaminoglycans predict outcomes in septic shock and acute respiratory distress syndrome. Am J Respir Crit Care Med. 2016;194(4):439-49.

29. Sun X, Li L, Overdier KH, Ammons LA, Douglas IS, Burlew CC, Zhang F, Schmidt EP, Chi L, Linhardt RJ. Analysis of total human urinary glycosaminoglycan disaccharides by liquid chromatography-tandem mass spectrometry. Anal Chem. 2015;87(12):6220-7.

30. Yang Y, Haeger SM, Suflita MA, Zhang F, Dailey KL, Colbert JF, Ford JA, Picon MA, Stearman RS, Lin L, et al. Fibroblast growth factor signaling mediates pulmonary endothelial Glycocalyx reconstitution. Am J Respir Cell Mol Biol. 2017;56(5):727-37

31. Haeger SM, Liu X, Han X, McNeil JB, Oshima K, McMurtry SA, Yang Y, Ouyang $Y$, Zhang F, Nozik-Grayck E, et al. Epithelial Heparan sulfate contributes to alveolar barrier function and is shed during lung injury. Am J Respir Cell Mol Biol. 2018;59(3):363-74.

32. Nelson A, Berkestedt I, Bodelsson M. Circulating glycosaminoglycan species in septic shock. Acta Anaesthesiol Scand. 2014;58(1):36-43.

33. Witthaut R, Busch C, Fraunberger P, Walli A, Seidel D, Pilz G, Stuttmann R, Speichermann N, Verner L, Werdan K. Plasma atrial natriuretic peptide and brain natriuretic peptide are increased in septic shock: impact of interleukin6 and sepsis-associated left ventricular dysfunction. Intensive Care Med. 2003;29(10):1696-702.

34. Kang H, Duran CL, Abbey CA, Kaunas RR, Bayless KJ. Fluid shear stress promotes proprotein convertase-dependent activation of MT1-MMP. Biochem Biophys Res Commun. 2015;460(3):596-602.

35. Platt MO, Ankeny RF, Jo H. Laminar shear stress inhibits cathepsin L activity in endothelial cells. Arterioscler Thromb Vasc Biol. 2006;26(8):1784-90.

36. van Haren FM, Sleigh J, Cursons R, La Pine M, Pickkers P, van der Hoeven $J G$. The effects of hypertonic fluid administration on the gene expression of inflammatory mediators in circulating leucocytes in patients with septic shock: a preliminary study. Ann Intensive Care. 2011;1(1):44.

37. Rhee $P$, Wang D, Ruff $P$, Austin B, DeBraux S, Wolcott K, Burris D, Ling G, Sun L. Human neutrophil activation and increased adhesion by various resuscitation fluids. Crit Care Med. 2000;28(1):74-8.

38. Suzuki K, Okada H, Takemura G, Takada C, Kuroda A, Yano H, Zaikokuji R, Morishita K, Tomita H, Oda K, et al. Neutrophil elastase damages the pulmonary endothelial Glycocalyx in lipopolysaccharide-induced experimental Endotoxemia. Am J Pathol. 2019. Epub ahead of print.

39. Semler MW, Self WH, Wanderer JP, Ehrenfeld JM, Wang L, Byrne DW, Stollings JL, Kumar AB, Hughes CG, Hernandez A, et al. Balanced crystalloids versus saline in critically ill adults. N Engl J Med. 2018;378(9):829-39.

40. Finfer S, McEvoy S, Bellomo R, McArthur C, Myburgh J, Norton R. Impact of albumin compared to saline on organ function and mortality of patients with severe sepsis. Intensive Care Med. 2011;37(1):86-96.

41. Torres Filho IP, Torres LN, Salgado C, Dubick MA. Plasma syndecan-1 and heparan sulfate correlate with microvascular glycocalyx degradation in hemorrhaged rats after different resuscitation fluids. Am J Phys Heart Circ Phys. 2016;310(11):H1468-78.

42. Murphy LS, Wickersham N, McNeil JB, Shaver CM, May AK, Bastarache JA, Ware LB. Endothelial glycocalyx degradation is more severe in patients with non-pulmonary sepsis compared to pulmonary sepsis and associates with risk of ARDS and other organ dysfunction. Ann Intensive Care. 2017;7(1):102.

43. Valerio L, Peters RJ, Zwinderman AH, Pinto-Sietsma SJ. Sublingual endothelial glycocalyx and atherosclerosis. A cross-sectional study. PLoS One. 2019;14(3):e0213097.

44. Chappell D, Jacob M, Hofmann-Kiefer K, Bruegger D, Rehm M, Conzen P, Welsch U, Becker BF. Hydrocortisone preserves the vascular barrier by protecting the endothelial glycocalyx. Anesthesiology. 2007;107(5):776-84.

45. Self WH, Semler MW, Bellomo R, Brown SM, deBoisblanc BP, Exline MC, Ginde AA, Grissom CK, Janz DR, Jones AE, et al. Liberal versus restrictive intravenous fluid therapy for early septic shock: rationale for a randomized trial. Ann Emerg Med. 2018;72(4):457-66.

\section{Publisher's Note}

Springer Nature remains neutral with regard to jurisdictional claims in published maps and institutional affiliations.
Ready to submit your research? Choose BMC and benefit from:

- fast, convenient online submission

- thorough peer review by experienced researchers in your field

- rapid publication on acceptance

- support for research data, including large and complex data types

- gold Open Access which fosters wider collaboration and increased citations

- maximum visibility for your research: over $100 \mathrm{M}$ website views per year

At BMC, research is always in progress.

Learn more biomedcentral.com/submissions 